\section{A Semi-Automatic Algorithm for Estimating Cobb Angle}

\author{
Safari A. ${ }^{1 \oplus}$, Parsaei H. ${ }^{1,2 * \odot}$, Zamani A. ${ }^{1}$, Pourabbas B. ${ }^{3}$
}

\begin{abstract}
Background: Scoliosis is the most common type of spinal deformity. A universal and standard method for evaluating scoliosis is Cobb angle measurement, but several studies have shown that there is intra- and inter- observer variation in measuring cobb angle manually.

Objective: Develop a computer- assisted system to decrease operator-dependent errors in Cobb angle measurement.

Methods: The spinal cord in the given $\mathrm{x}$-ray image of the spine is highlighted using contract-stretching technique. The overall structural curvature of the spine is determined by a semi-automatic algorithm aided by the operator. Once the morphologic curve of the spine is determined, in the last step the cobb-angle is estimated by calculating the angle between two normal lines to the spinal curve at the inflection points of the curve.
\end{abstract}

Results: Evaluation results of the developed algorithms using 14 radiographs of patients ( 4 - 40 years old) with cobb angle ranges from 34 - 82 degrees, revealed that the developed algorithm accurately estimated cobb angle. Statistical analysis showed that average angle values estimated using the developed method and that provided by experts are statistically equal. The correlation coefficient between the angle values estimated using the developed algorithm and those provided by the expert is 0.81 .

Conclusion: Compared with previous algorithms, the developed system is easy to use, less operator-dependent, accurate, and reliable. The obtained results are promising and show that the developed computer-based system could be used to quantify scoliosis by measuring Cobb angle.

Citation: Safari A, Parsaei H, Zamani A, Pourabbas B. A Semi-Automatic Algorithm for Estimating Cobb Angle. J Biomed Phys Eng. 2019;9(3):317-326. https://doi.org/10.31661/jbpe.v9i3Jun.730

\section{Keywords}

Cobb-angle Measurement, Curve-fitting, Scoliosis, Spinal Curvature Measurement

\section{Introduction}

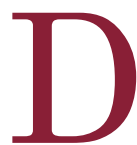
eformity of spine happens because of abnormal curvature of the spine, which can be detected as Kyphosis in sagittal plane. This deformity can cause Lordosis in the lumbar spine (lower back) or Kyphosis in the thoracic spine (upper back), or in some cases, scoliosis (side-to-side curvature). The most common type of deformity is known as scoliosis [1]. Scoliosis, in general, is defined as an abnormal side-to-side curvature of the spine to the vertical axis, which is depicted in Figure 1 [2].

It is not possible to examine and diagnose the value of Cobb-angle by merely using unaided eye, which is the reason of using medical imag-
${ }^{1}$ Department of Medical

Physics and Engineering,

School of Medicine, Shi-

raz University of Medical

Sciences, Shiraz, Iran

${ }^{2}$ Shiraz Neuroscience

Research Center, Shiraz

University of Medical Sci-

ences, Shiraz, Iran

${ }^{3}$ Bone and Joint Dis-

eases Research Center,

Department of Orthope-

dic Surgery, Chamran

Hospital, Shiraz Univer-

sity of Medical Sciences,

Shiraz, Iran

*Corresponding author:

H. Parsaei,

Department of Medical

Physics and Engineer-

ing, Shiraz University

of Medical Sciences,

Shiraz, Iran

E-mail: hparsaei@sums.

ac.ir

Received: 11 February 2107

Accepted: 4 July 2017 


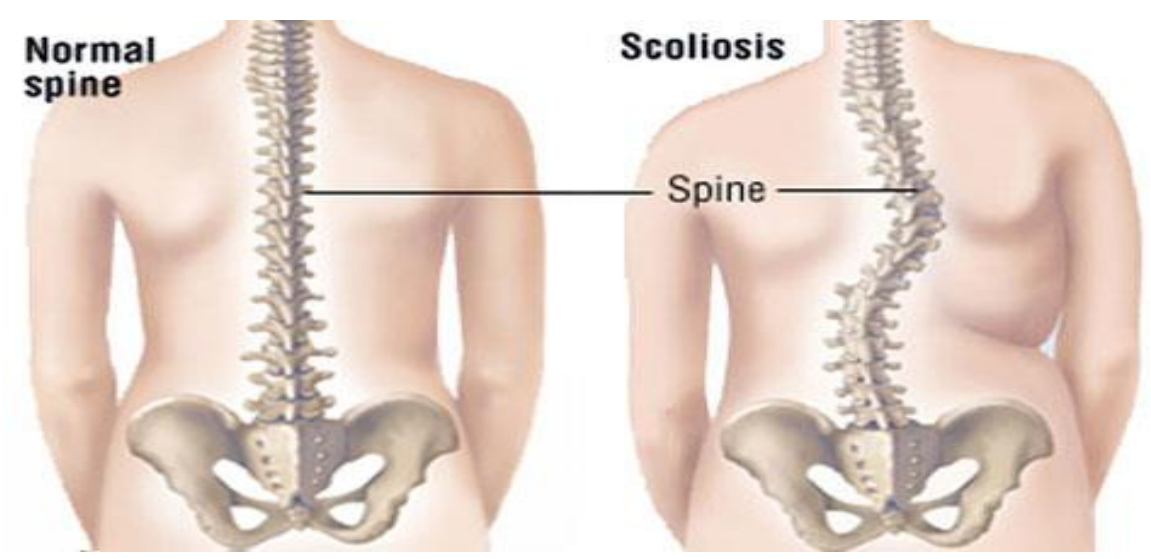

Figure 1: Comparison of a healthy patient with one with scoliosis (available from: http://www. sierraneurosurgery.com/procedures-and-expertise/adult-scoliosis/.)

ing in both diagnosis and progression of the disease before and after treatment. X-ray radiography is a standard method for the diagnosis of scoliosis. In order to measure the value of spinal deformity, an Anterior-Posterior radiographic image of the vertebral column is taken, which shows the sideway spinal curvature [1]. The most accepted index for evaluating scoliosis is measuring the Cobb-angle from images taken by radiography [3].

Cobb-angle is also employed in quantitative evaluation of spinal deformity progression. Generally, any increament greater than 5 degrees is considered as progression of spinal deformity [4]. Cobb-angle measurement techniques include choosing the most deviated vertebra in the upper back, the most deviated one in the lower back, and then drawing the tangent lines to these two vertebrae. The angle formed by intersection of these lines is the Cobb-angle. If continuing the lines is difficult, the angle can be formed by lines perpendicular to the mentioned tangent lines, this procedure is illustrated in Figure 2.

In Cobb-angle measurement, which is performed by a ruler, a pencil and a protractor, one can draw tangent lines along the end of vertebrae on X-ray images, and then measure the angle using a protractor [5]. This procedure

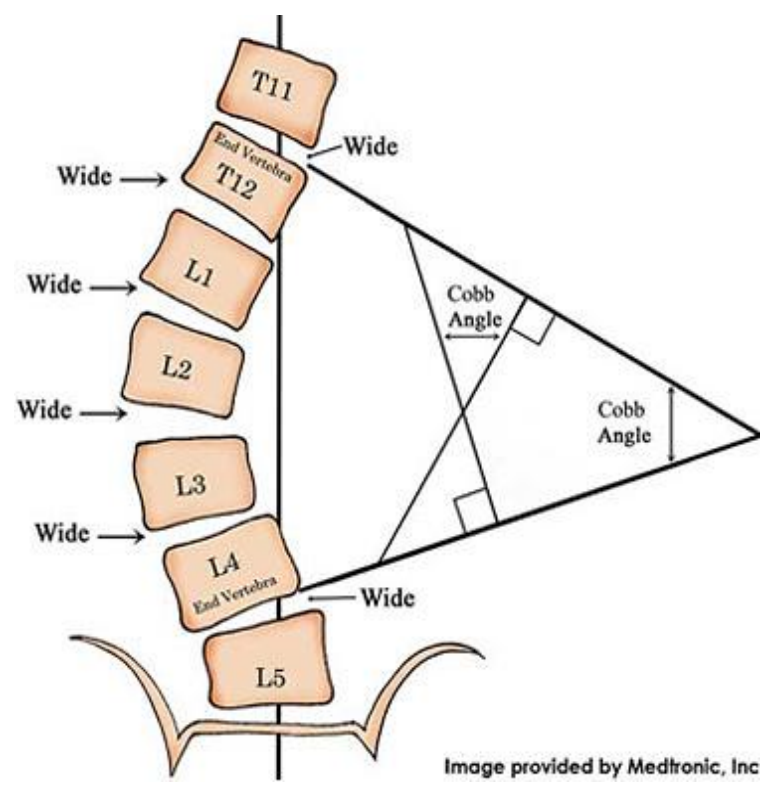

Figure 2: Manual method for measuring the Cobb-angle (available from: https://www. spinemd.com/news-philanthropy/scoliosismeasurements).

highly dependents on the user's experience and judgment. The aforementioned method is widely applied for measuring the Cobb-angle $[6,7]$, but there are always varaibility in the reported values. It has been reported that if the most deviated vertebrae have not been chosen 
in advance, the mean variation would be about 7.2 degrees, and if the most deviated vertebrae have been chosen before the measurement, the mean variation would be 6.3 degrees approximately [8].

Measurement errors originate from choosing different vertebrae as well as differences in estimating the slopes of the vertebrae. Even if the same vertebrae are selected as the most deviated ones, a standard error of 3-5 degrees can be observed (Inter-observer). However, if two individuals measure the Cobb-angle under the same conditions (Intra-observer), a standard error of 5-7 degrees can be expected $[4,9]$. Being exhausting and time-consuming [10], the manual method should be modified to be simple and accurate.

Technological advancements have been increased due to the usage of digital X-ray imaging for clinical purposes, and various algorithms have been proposed for measuring the Cobb-angle [6]. In 1980, Barray et al. proposed a method using mathematical equations for calculating the spinal curvature [11]. First, some points are chosen on the vertebral column, and a polynomial curve is estimated. Then, in view of the fact that the most deviated vertebrae have the widest angle to the horizontal line through apex; the points of interest can be calculated using differential calculus. Apex is a vertebra of the longest distance from Sacrum Central Vertebra Line (SCVL), which is a vertical line drawn from the center of sacrum upwards. Critical points of the curve are points, in which the curvature of a curve is changed. It can be proved that the most deviated vertebrae are the critical points of the curve, at which the second derivative equals zero. After intersecting those vertical lines with tangent ones, there would be an angle which is called the Cobb-angle.

In 1998, Kevin et al., drew a comparison between computer-aided methods using digital radiographic images, and the manual method [12], which was based on the studies of Morrissy and Carman $[8,13]$. Using orthographic software, separated lines were drawn to the last up and down vertebrae, and software would automatically calculate the angle between the lines. In 2002, Chockalingam et al. presented a technique for Cobb-angle measurement using Matlab software [14]. In this program, the radiographic image depicted horizontally, and the user can choose a proper region of vertebral column for measurement. The two points selected by the computer mouse are located on the right and left of the top of the radiographic image. Next, the image is divided into 8 sections, and the observer chooses 2 points on each line, where the anatomical medial and lateral edges of the vertebra cross the line. The software chooses the midpoint of the line crossing the selected points, and connects them which form the spinal midline. Finally, the widest angle is calculated and the Cobbangle is identified.

In 2005, Micheal et al., studied the measurement of Cobb-angle using a new device called Oxford Cobbometer [15], which presented a manual method, and then they compared this method with traditional goniometers. In 2007, Gstoettner et al. introduced a method using Icoview software [5]. The user draws lines to the most deviated upper and lower vertebrae, and the program calculates the Cobb-angle automatically. In 2008, Allen et al. suggested an automatic method for measuring the Cobbangle [6], which was based on the active shape model approach, but required training. Images with Cobb-angles of 20-50 degrees, which do not need surgery, are used for training. Cobbangles are calculated automatically using the best curved fitted on the tangent lines on the last known vertebra. In 2009, Zhang et al. proposed a novel approach, which was founded on contrast dark and light adaption, and choosing the Region of Interest (ROI) [16]. The method takes advantage of Canny technique for the identification of vertebra edges, as well as fuzzy Hough transformation in finding the slope of tangent lines on the last vertebra, and finally, measures the Cobb-angle by calculat- 
ing the angle formed by continuing these lines.

In 2009, Omoto et al. estimated the Cobbangle using a 4-th order polynomial curve fitting on the spinal curvature [17]. In order to smooth the image, first, the image undergoes a pre-processing using Gaussian filter with $\sigma=24$, and mask size of $3 \times 3$. The method is applied to the whole vertebral column, and the midpoint of sacrum's plate is considered as zero point. Therefore, point 1 is located on the right of the superior endplate, point 2 underneath point 1 , point 3 on the left of the superior endplate and finally, point 4 underneath point 3 . Connecting these 4 points, point 5 is obtained, which is the center of the vertebra. After that, a curve of 4-th order would be fitted for these 5 points, and then, the $\mathrm{x}$-axis and $y$-axis should be changed. After calculating the inflation point using second derivative, the tangent line on the curve, and then the perpendicular line on the tangent line would be drawn. The angle between these lines, which can be obtained from the tangent equations, is the Cobb-angle.

In 2010, Tanure et al. suggested a technique using Matlab software [18], which benefitted from both manual as well as automatic methods. In this computer-assisted approach, two points on the superior endplates of the first vertebra, and two other points on the inferior endplates of the last vertebra are chosen, then connected, and lastly, the Cobb-angle is calculated as the angle between these lines. Therefore, there are points on the curvature. All steps of the procedure are accomplished by software, and the user just has to identify the points. The software also offers tools for increasing the image contrast, silhouette en- hancement and negative image effect. In 2002, Kundu et al. introduced a computer-assisted method which only required the most deviated vertebra [19]. The rest of the proposed process was completed using image processing techniques based on a 1-st order Gaussian function of a novel algorithm, used in identifying the horizontal edges.

As it is obvious, most of recent studies are sensitive to choosing the proper vertebrae which can be a source of measurement error. The presented article addresses this source of measurement error.

\section{Material and Methods}

In this work we used $14 \mathrm{X}$-ray radiographic images from Picture Archiving Communication System (PACS) from Shahid Chamran Hospital, Shiraz, Iran. Patients are between ages 4 and 40, and Cobb-angles are between 34 and 82 degrees. Due to blurriness of the images, first, the images undergo an X-ray preprocessing. Then, some points are chosen by the user on the vertebral column, and a 5-th order polynomial curve are fitted for the points. Having calculated the inflection points and drawn the lines perpendicular to the curve at these points, the angles between each two perpendicular lines are measured, and the widest angle is considered as the Cobb-angle. Different phases of the procedure are illustrated in a block diagram in Figure 3.

This paper measures the Cobb-angle using both manual and semi-automatic approaches, and compares all obtained results accurately. In the manual method, the measurements have been done by two specialists, the values reported by the more experienced one is consid-

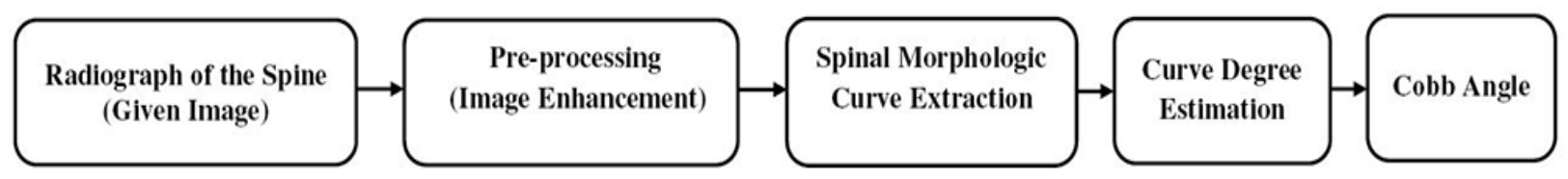

Figure 3: Phases of Cobb-angle estimation 
ered as the Gold Standard, and the other measurement is considered as the manual method measurement. The semi-automatic technique is performed by the proposed algorithm in the article.

\section{Image Pre-processing}

Radiographic images are noisy and need one stage of pre-processing to increase the quality of anatomical structure of the image [20]. Sometimes, the edge of the vertebral column overlaps with the ribcage and inner organs such as the heart or lungs, and causes blurriness in the images. Thus, the thin edges may disappear in X-ray, and the detection would be an incredibly difficult. Therefore, it is mandatory to perform the pre-processing to reduce noise in images [21]. This work benefits a method for image processing which is relevant to image pixels and processes the brightness. Brightness transformation, which has impacts on singular pixels of image, is used in contrast stretching. The presented work takes advantage of contrast stretching on brightness in [0, L-1], where $L$ is the maximum level of brightness, which is 256 in this work. The pixel values in the given image are converted to new values using the transformation function shown in Figure 4. In this work we used the following settings, $\left(r_{1}, s_{1}\right)=\left(r_{\min }, 0\right)$ and $\left(r_{2}\right.$, $\left.s_{2}\right)=\left(r_{\max }, L-1\right)$; where $r_{\min }$ and $r_{\max }$ represent the minimum and maximum gray levels in the image under analysis, respectively [22]. The value for $L$ as mentioned above is set to 256 .

As the result of this process, white regions look whiter and dark regions seem darker as presented in Figure 5.

\section{Spinal Morphological Curve Extrac- tion}

First, several points are chosen on the spinal curvature by a user. The user is asked to choose those points according to the overall shape of the spine. The number of points should be equal or greater than the number of vertebrae. It is worth pointing out that here

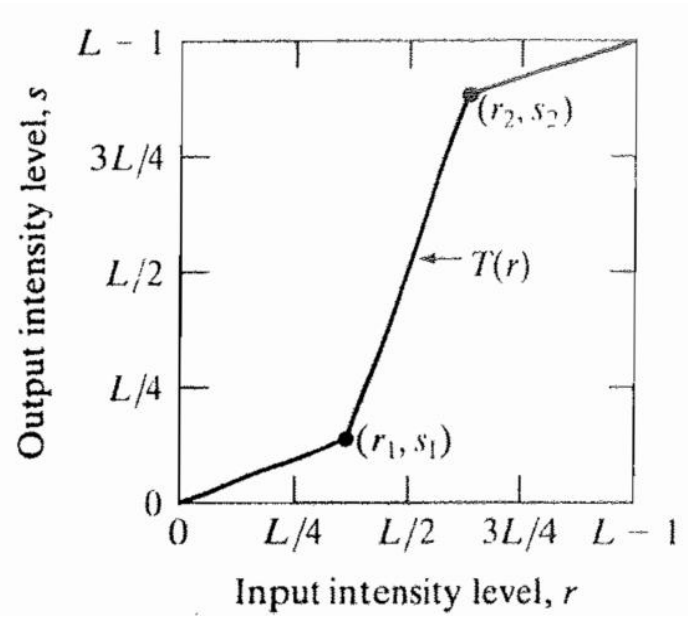

Figure 4: Applying piecewise linear transformation functions to image brightness [22].

just choosing at least one point for each vertebra is enough regardless of the position of the point on the corresponding vertebra. The point identified for each vertebra should be located on the vertebrae, but it is not required that the chosen point be the center of vertebra. Finding the center of vertebra is difficult in lowcontrast images, and is highly dependent on the operator experience and skill. Therefore, the flexibility provided in our algorithm makes the system robust and easy to use. In addition, it provides this opportunity to the user to use more points and ultimately decrease the estimation error of the developed Cobb angle estimation algorithm. Finally, a 5-th order polynomial curve is fitted to the identified points.

Curve fitting on the coordinates of selected columns and rows is done separately, according to the point number, that is, the point number is considered as an independent parameter, and the coordinates of row/column are considered as a dependent parameter, which identifies the number of points required for the curve fitting by user. In fact, there are two fitted curves; one includes the number of vertebral to x-curve $(x(n))$, and the other is the number of vertebral to y-curve $(y(n))$. In this case, the fitted curve is of more complexity in 


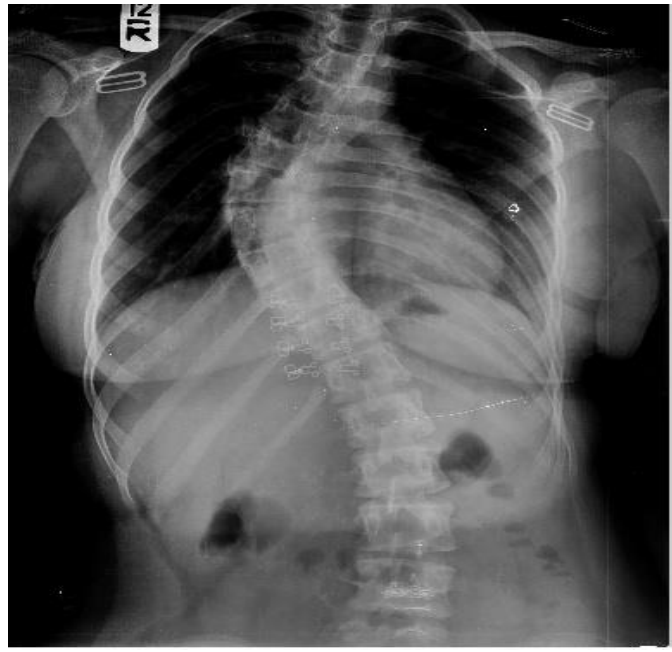

a

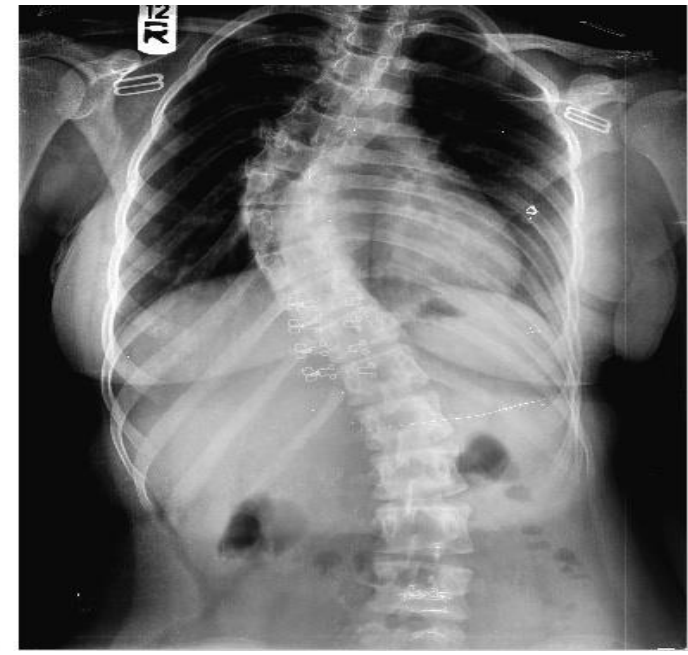

b

Figure 5: a) original image (left), b) processed image (right)

mathematical terms, but one can make sure that its functional properties are guaranteed under all circumstances. As a result, equation 1 is used to calculate the derivative of $y$ with respect to $x$. Figure 6 shows the curve fitting.

$$
\frac{d y}{d x}=\frac{d y}{d n} \times \frac{d n}{d x}
$$

\section{Curve Degree Estimation}

The next step is calculating the inflection points of the curves, which reveals the most deviated vertebrae. Finding the most deviated points automatically has also the benefit that there is no need for the specialist or user to identify these vertebrae which leads to error reduction.

The last phase is estimating the Cobb-angle. The lines perpendicular (normal) to the spinal curve at the inflection points should be drawn, and having them intersected, the widest angle, which can be calculated from tangent equation (equation 2), is the Cobb-angle. Figure 7 outlines this process. In equation 2, $\theta$ is the angle between the lines, and $m$ and $\mathrm{m}$ ' $m$ ' are slopes of the lines, respectively.

$$
\theta=\tan ^{-1}\left|\frac{m-m^{\prime}}{1+m m^{\prime}}\right|
$$

\section{Result and Discussion}

The measurement results, also compared

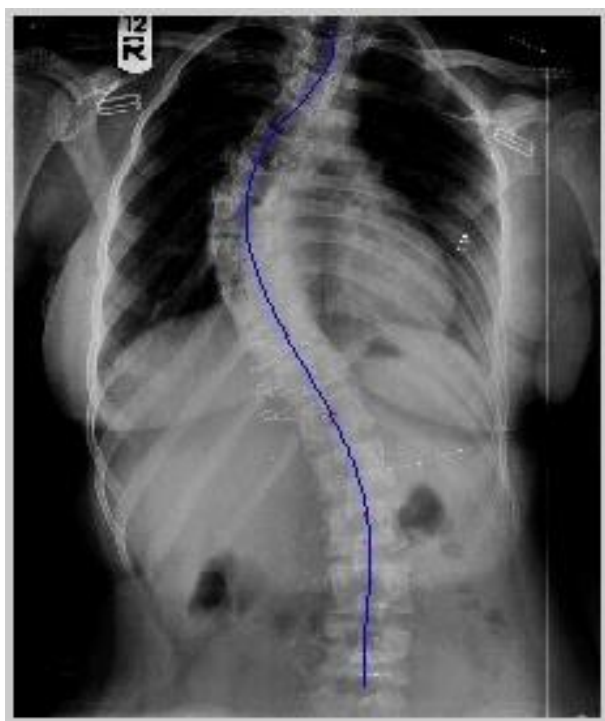

Figure 6: Example of a 5-th order polynomial curve fitting for the spinal curvature 


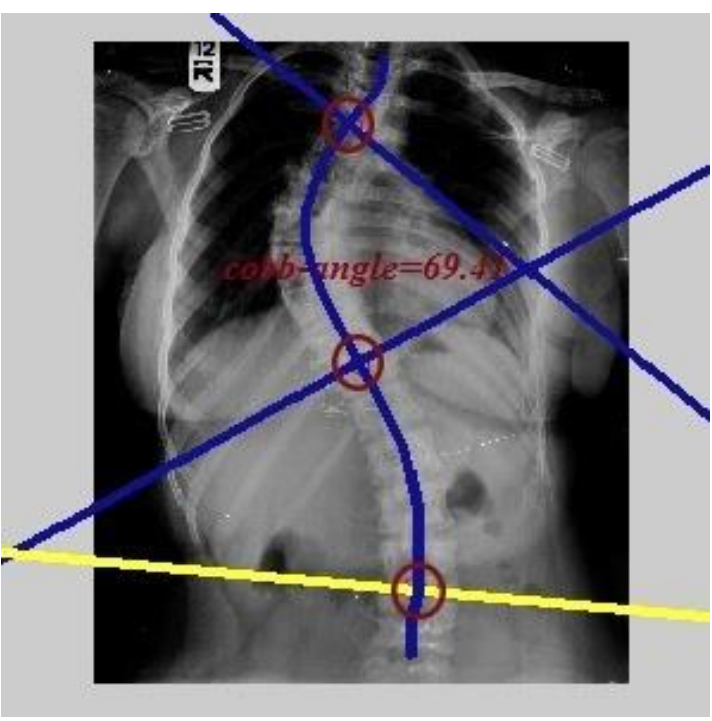

Figure 7: Cobb-angle measurement using tangent equations

with Omoto results [17], are summerized in Table 1. As it was mentioned before, manual measurement column is the result of measuring Cobb-angle by a specialist, and Omoto column exhibits the measurement results evaluated by the method proposed by Omoto et al. [17]. The numbers in the first column were calculated by an experienced specialist and considered as gold standard. All numbers are the average of two measurements obtained by repeating the experiments two times.

As it can be seen, the angles measured by the proposed semi-automatic method are greater than those of gold standard and manual method, but the differences with the gold standard are small. It is clear that the proposed algorithm is more accurate than the Omoto method which is proved by the values in the table (see results for $\mathrm{C} 5$ as an example). Another advantage of the algorithm is that there are smaller differences between the measurements themselves which shows the higher accuracy of the presented method than that of manual method. It is because of the fact that choosing the most deviated vertebrae is less dependent on the user's judgment. Moreover, it should be mentioned that although the algorithm is semi-

Table 1: Comparison between Cobb-angle measurement and gold standard.

\begin{tabular}{ccccc} 
Case & Gold standard & Proposed method & Omoto method [17] & Manual measurement \\
\hline C1 & 46.0 & 47.2 & 58.2 & 46.0 \\
\hline C2 & 81.0 & 82.3 & 82.5 & 88.0 \\
\hline C3 & 56.0 & 61.0 & 63.7 & 51.5 \\
\hline C4 & 82.0 & 85.8 & 82.2 & 85.0 \\
\hline C5 & 55.0 & 56.0 & 76.4 & 57.5 \\
\hline C6 & 81.0 & 81.5 & 80.9 & 84.0 \\
\hline C7 & 45.0 & 49.7 & 26.1 & 45.0 \\
\hline C8 & 52.0 & 51.7 & 51.1 & 52.0 \\
\hline C9 & 34.0 & 35.9 & 40.6 & 35.0 \\
\hline C10 & 38.0 & 39.1 & 49.9 & 36.0 \\
\hline C11 & 74.0 & 72.6 & 73.7 & 68.0 \\
\hline C12 & 73.0 & 78.9 & 81.3 & 66.0 \\
\hline C13 & 81.0 & 81.6 & 86.1 & 80.0 \\
\hline C14 & 72.0 & 72.2 & 72.2 & 67.5 \\
\hline Mean & 62.1 & 63.9 & 66.1 & 61.5 \\
\hline STD & 16.8 & 16.7 & 17.7 & 17.5 \\
\hline
\end{tabular}


automatic, it takes less time to find the Cobbangle, since there is no need to find the center of each vertebra.

Omoto et al. [17] by considering the vertebral edges and connecting them found centers of vertebrae, and after that, fitted a 4-th order curve. However, in the presented work, a 5-th order curve is fitted and there is no need to find centers of vertebrae which is a time-consuming task. In addition, it should be stressed out that in some cases such as the one illustrated in Figure 8, the Omoto method may not even obtain accurate results. It is due to the fact that in such cases there are more than one $y$ value for a specific $x$ value. In other words, the property of being a function was not necessarily considered in the Omoto method, while the presented algorithm solves this problem through using the point number as an independent variable.

\section{Conclusion}

In this research, after pre-processing x-ray radiographic images, Cobb-angles are measured using a 5-th order polynomial curve fitting. Although the method is semi-automatic because of choosing points by a user, there is no need to calculate the center of the vertebra which leads to make the algorithm fast and less dependent on the operator skill. Moreover, compared to manual and Omoto methods, this approach is more accurate. In addition, in case of several $y$ values for one specific $x$ value, the proposed algorithm is more reliable thanks to using point numbers as independent variables.

\section{Acknowledgment}

The authors would like to express their sincere appreciation to Shahid Chamran Hospital in Shiraz, Iran, for preparing the radiographic images. The paper has been extracted from parts of the M.Sc. thesis by Ameneh Safari supported by the Research Council of Shiraz University of Medical Sciences (94-01-0111021).

\section{Conflict of Interest}

None

\section{References}

1. Adam C, Dougherty G. Applications of medical image processing in the diagnosis and treatment of spinal deformity. Medical Image Processing: Springer; 2011. p. 227-48.

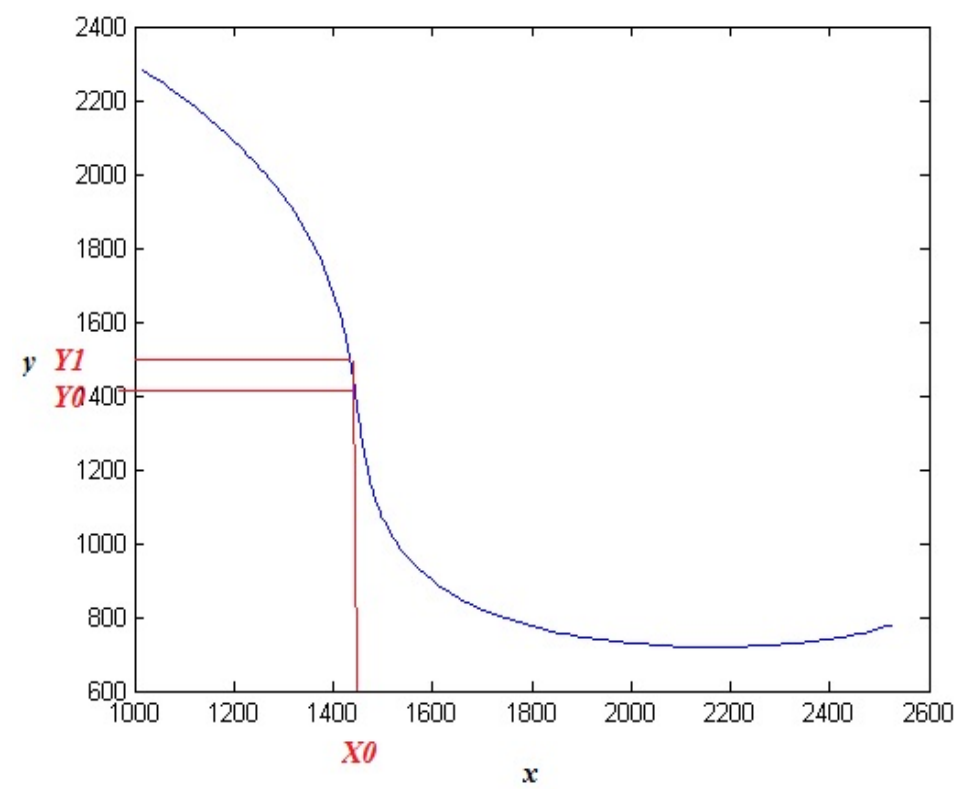

Figure 8: Type of spinal curvature: more than one $y$ for one $x$ 
2. Herring JA. Tachdjian's Pediatric Orthopaedics EBook: From the Texas Scottish Rite Hospital for Children: Elsevier Health Sciences; 2013.

3. Asher MA, Strippgen WE, Heinig CF, Carson WL. Isola spinal implant system. Semin Spine Surg. 1992;4:175-81.

4. Jaeger U, Koenig R, Gieseke J, Wagner U, Kandyba $\mathrm{J}$, Ostertun B, editors. MR total spine projection in juvenile scoliosis: an alternative to radiographic follow-up. Radiology; 1998: RADIOLOGICAL SOC NORTH AMER 20TH AND NORTHAMPTON STS, EASTON, PA 18042 USA.

5. Gstoettner M, Sekyra K, Walochnik N, Winter P, Wachter R, Bach CM. Inter- and intraobserver reliability assessment of the Cobb angle: manual versus digital measurement tools. Eur Spine $\mathrm{J}$. 2007;16:1587-92. doi.org/10.1007/s00586-0070401-3. PubMed PMID: 17549526. PubMed PMCID: 2078306.

6. Allen S, Parent E, Khorasani M, Hill DL, Lou E, Raso JV. Validity and reliability of active shape models for the estimation of cobb angle in patients with adolescent idiopathic scoliosis. J Digit Imaging. 2008;21:208-18. doi.org/10.1007/s10278007-9026-7. PubMed PMID: 17340228. PubMed PMCID: 3043859.

7. Kuklo TR, Potter BK, Polly DW, Jr., O'Brien MF, Schroeder TM, Lenke LG. Reliability analysis for manual adolescent idiopathic scoliosis measurements. Spine (Phila Pa 1976). 2005;30:444-54. doi.org/10.1097/01.brs.0000153702.99342.9c. PubMed PMID: 15706343.

8. Morrissy RT, Goldsmith GS, Hall EC, Kehl D, Cowie $\mathrm{GH}$. Measurement of the Cobb angle on radiographs of patients who have scoliosis. Evaluation of intrinsic error. J Bone Joint Surg Am. 1990;72:320-7. doi.org/10.2106/00004623-199072030-00002. PubMed PMID: 2312527.

9. Konig R, Jaeger U, Ostertun B, Kandyba J, Wagner U, Gieseke J, et al. MR whole-spine recording: computer-assisted simulation of the conventional x-ray technic. Rofo. 1999;170:258-61. doi. org/10.1055/s-2007-1011037. PubMed PMID: 10230434

10. Kundu R, Chakrabarti A, Lenka PK. Cobb angle measurement of scoliosis with reduced variability. arXiv preprint arXiv:1211.5355. 2012.

11. Jeffries BF, Tarlton M, De Smet AA, Dwyer SJ, 3rd, Brower AC. Computerized measurement and analysis of scoliosis: a more accurate representation of the shape of the curve. Radiology. 1980;134:381-
5. doi.org/10.1148/radiology.134.2.6986054 PubMed PMID: 6986054.

12. Shea KG, Stevens PM, Nelson M, Smith JT, Masters KS, Yandow S. A comparison of manual versus computer-assisted radiographic measurement. Intraobserver measurement variability for Cobb angles. Spine (Phila Pa 1976). 1998;23:551-5. doi.org/10.1097/00007632-199803010-00007. PubMed PMID: 9530786.

13. Carman DL, Browne RH, Birch JG. Measurement of scoliosis and kyphosis radiographs. Intraobserver and interobserver variation. J Bone Joint Surg Am. 1990;72:328-33. doi.org/10.2106/00004623199072030-00003. PubMed PMID: 2312528.

14. Chockalingam N, Dangerfield PH, Giakas G, Cochrane T, Dorgan JC. Computer-assisted Cobb measurement of scoliosis. Eur Spine J. 2002;11:3537. doi.org/10.1007/s00586-002-0386-x. PubMed PMID: 12193997. PubMed PMCID: 3610477.

15. Rosenfeldt MP, Harding IJ, Hauptfleisch JT, Fairbank JT. A comparison of traditional protractor versus Oxford Cobbometer radiographic measurement: intraobserver measurement variability for Cobb angles. Spine (Phila Pa 1976). 2005;30:4403. doi.org/10.1097/01.brs.0000153401.78638.cb. PubMed PMID: 15706342

16. Zhang J, Lou E, Le LH, Hill DL, Raso JV, Wang Y. Automatic Cobb measurement of scoliosis based on fuzzy Hough Transform with vertebral shape prior. J Digit Imaging. 2009;22:463-72. doi. org/10.1007/s10278-008-9127-y. PubMed PMID: 18516643. PubMed PMCID: 3043716.

17. Omoto E, Wakamatsu O, Sanada S, Tokyo JP, Kanazawa JP. Development of software for automatic measurement of Cobb angle and quantitative assessment method for followup in radiographs of patients with scoliosis. European Society of Radiology. Vienna: Austria; 2009.

18. Tanure MC, Pinheiro AP, Oliveira AS. Reliability assessment of Cobb angle measurements using manual and digital methods. Spine J. 2010;10:769-74. doi.org/10.1016/j.spinee.2010.02.020. PubMed PMID: 20359959.

19. Kundu R, Lenka P, Kumar R, Chakrabarti A. Cobb angle quantification for scoliosis using image processing techniques. Proceedings of the International Journal of Computer Applications. 2012:611.

20. Behrenbruch CP, Petroudi S, Bond S, Declerck JD, Leong FJ, Brady JM. Image filtering techniques for medical image post-processing: an over- 
view. $\mathrm{Br} J$ Radiol. 2004;77 Spec No 2:S126-32. doi.org/10.1259/bjr/17464219. PubMed PMID: 15677354.

21. Duong L, Cheriet F, Labelle H. Automatic detection of scoliotic curves in posteroanterior radiographs.
IEEE Trans Biomed Eng. 2010;57:1143-51. doi. org/10.1109/TBME.2009.2037214. PubMed PMID: 20142161.

22. Gonzalez RC, Woods RE. Digital Image Processing. 4th edition. New York, NY: Pearson; 2017. 